\title{
On unitary equivalence of invariant subspaces of the Dirichlet space
}

\author{
by \\ Kunyu Guo (Shanghai) and Liankuo Zhao (Linfen)
}

\begin{abstract}
It is shown that in the Dirichlet space $\mathcal{D}$, two invariant subspaces $\mathcal{M}_{1}$, $\mathcal{M}_{2}$ of the Dirichlet shift $M_{z}$ are unitarily equivalent only if $\mathcal{M}_{1}=\mathcal{M}_{2}$.
\end{abstract}

1. Introduction. Let $\mathbb{D}$ be the open unit disk, and $d A$ the normalized Lebesgue measure on $\mathbb{D}$. The Dirichlet space $\mathcal{D}$ consists of the analytic functions $f$ on $\mathbb{D}$ with finite Dirichlet integral

$$
D(f)=\int_{\mathbb{D}}\left|f^{\prime}(z)\right|^{2} d A(z) .
$$

It is easy to verify that $\mathcal{D} \subset H^{2}$, the Hardy space on $\mathbb{D}$.

Endow $\mathcal{D}$ with norm $\|\cdot\|$,

$$
\|f\|^{2}=\|f\|_{2}^{2}+D(f), \quad f \in \mathcal{D}
$$

where $\|f\|_{2}$ is the norm of $f$ in $H^{2}$. Then $\mathcal{D}$ is a reproducing kernel function space with reproducing kernel

$$
K_{\lambda}(z)=\frac{1}{\bar{\lambda} z} \log \frac{1}{1-\bar{\lambda} z}, \quad \lambda, z \in \mathbb{D} .
$$

It is well known that $K_{\lambda}$ is a complete Nevanlinna-Pick kernel.

Let $M_{z}$ be the operator of multiplication by $\varphi(z)=z$ on $\mathcal{D}$, called the Dirichlet shift. It is an important operator on $\mathcal{D}$ which has been extensively studied, and there is a large literature concerning invariant subspaces of $M_{z}$ ([5], 6], [7, [8]-[11]). We refer the readers to the survey paper [12] for more information about the Dirichlet space $\mathcal{D}$ and the Dirichlet shift $M_{z}$.

Let $\operatorname{Lat}\left(M_{z}, \mathcal{D},\|\cdot\|\right)$ be the lattice of invariant subspaces of $M_{z}$ in $\mathcal{D}$. Recall that two invariant subspaces $\mathcal{M}_{1}$ and $\mathcal{M}_{2}$ of $M_{z}$ are unitarily equivalent if there exists a unitary operator $U: \mathcal{M}_{1} \rightarrow \mathcal{M}_{2}$ such that $\left.U M_{z}\right|_{\mathcal{M}_{1}}=$ $\left.M_{z}\right|_{\mathcal{M}_{2}} U$. 
In [7], unitary equivalence of invariant subspaces of $M_{z}$ was studied, and it was shown that if two invariant subspaces $\mathcal{M}_{1}, \mathcal{M}_{2}$ satisfy one of the following conditions:

1. $\mathcal{M}_{1}$ contains an outer function,

2. $\mathcal{M}_{1} \subseteq \mathcal{M}_{2}$,

then $\mathcal{M}_{1}$ and $\mathcal{M}_{2}$ are unitarily equivalent if and only if $\mathcal{M}_{1}=\mathcal{M}_{2}$ [7, Theorem 2].

In this paper, by using some ideas of [11, we prove the following theorem.

Theorem 1.1. Suppose $\mathcal{M}_{1}, \mathcal{M}_{2} \in \operatorname{Lat}\left(M_{z}, \mathcal{D},\|\cdot\|\right)$ are unitarily equivalent. Then $\mathcal{M}_{1}=\mathcal{M}_{2}$.

Also, endow $\mathcal{D}$ with the equivalent norm $\|\cdot\|_{0}$,

$$
\|f\|_{0}^{2}=|f(0)|^{2}+D(f), \quad f \in \mathcal{D} .
$$

Then the reproducing kernel of $\mathcal{D}$ is

$$
K_{\lambda}^{0}(z)=1+\log \frac{1}{1-\bar{\lambda} z}, \quad \lambda, z \in \mathbb{D} .
$$

With the norm $\|\cdot\|_{0}$ on $\mathcal{D}$, we will show the following theorem.

Theorem 1.2. Let $\mathcal{M}_{1}, \mathcal{M}_{2} \in \operatorname{Lat}\left(M_{z}, \mathcal{D},\|\cdot\|_{0}\right)$. Then they are unitarily equivalent if and only if they are equal.

Let $H^{1,2}(\mathbb{D})$ be the completion of

$$
\left\{u \in C^{1}(\mathbb{D}):\|u\|_{1,2}=\left(\int_{\mathbb{D}}|u|^{2} d A+\int_{\mathbb{D}}\left(\left|\frac{\partial u}{\partial z}\right|^{2}+\left|\frac{\partial u}{\partial \bar{z}}\right|^{2}\right) d A\right)^{1 / 2}<\infty\right\}
$$

with respect to the Sobolev norm $\|\cdot\|_{1,2}$ (see [1]). Then the Dirichlet space $\mathcal{D}$ is a closed subspace of $H^{1,2}(\mathbb{D})$ with the norm $\|\cdot\|_{1}$,

$$
\|f\|_{1}^{2}=\int_{\mathbb{D}}|f(z)|^{2} d A(z)+D(f), \quad f \in \mathcal{D} .
$$

We have the following theorem.

Theorem 1.3. Let $\mathcal{M}_{1}, \mathcal{M}_{2} \in \operatorname{Lat}\left(M_{z}, \mathcal{D},\|\cdot\|_{1}\right)$. Then they are unitarily equivalent if and only if they are equal.

2. Unitary equivalence of invariant subspaces in the norm $\|\cdot\|$. In this section, the norm $\|\cdot\|$ on $\mathcal{D}$ is defined as

$$
\|f\|^{2}=\|f\|_{2}^{2}+D(f), \quad f \in \mathcal{D},
$$

where $\|f\|_{2}$ is the norm of $f$ in $H^{2}$, and $\langle\cdot, \cdot\rangle$ is the corresponding inner product on $\mathcal{D}$.

First, we fix some notation and cite some results about invariant subspaces of $M_{z}$ in $\mathcal{D}$. 
For a set $S \subset \mathcal{D}$, let $[S]$ denote the invariant subspace of the Dirichlet shift $M_{z}$ generated by $S$. Let $\mathbb{T}=\partial \mathbb{D}$ be the unit circle, and $d m$ the normalized Lebesgue measure on $\mathbb{T}$. We need the following results.

Theorem 2.1. Let $\mathcal{M} \in \operatorname{Lat}\left(M_{z}, \mathcal{D},\|\cdot\|\right)$. Then

(i) [8, Theorem 2(c)] $\mathcal{M} \ominus z \mathcal{M}$ is one-dimensional.

(ii) [5, Theorem 1] $[\mathcal{M} \ominus z \mathcal{M}]=\mathcal{M}$.

Theorem 2.2. Let $\mathcal{M} \in \operatorname{Lat}\left(M_{z}, \mathcal{D},\|\cdot\|\right)$. If $\phi \in \mathcal{M} \ominus z \mathcal{M}$, then

(i) [10, Theorem 3.1] $\phi$ is a multiplier of $\mathcal{D}$.

(ii) [11, Theorem $2.2(\mathrm{a})] \phi^{\prime} \in N(\mathbb{D})$, the Nevanlinna class of $\mathbb{D}$.

From Theorem 2.2(ii) and [4, Theorem 5.3], if $\phi \in \mathcal{M} \ominus z \mathcal{M}$, then for a.e. $\xi \in \mathbb{T}$, the nontangential limit of $\phi^{\prime}$ at $\xi, \phi^{\prime}(\xi)$, exists. For $f \in \mathcal{D}$ and $\xi \in \mathbb{T}$, let $f(\xi)$ be the nontangential limit of $f$ at $\xi$.

The following lemma comes essentially from the proof of [7, Lemma 2], which gives a necessary condition for two invariant subspaces to be unitarily equivalent.

Lemma 2.3. Let $\mathcal{M}_{1}, \mathcal{M}_{2} \in \operatorname{Lat}\left(M_{z}, \mathcal{D},\|\cdot\|\right)$ and $U: \mathcal{M}_{1} \rightarrow \mathcal{M}_{2}$ be a unitary operator such that $\left.U M_{z}\right|_{\mathcal{M}_{1}}=\left.M_{z}\right|_{\mathcal{M}_{2}} U$. Then for any $f \in \mathcal{M}_{1}$ and $g=U f,|f(\xi)|^{2}=|g(\xi)|^{2}$, a.e. $\xi \in \mathbb{T}$.

For $f \in \mathcal{D}$ and $\xi \in \mathbb{T}$, define the local Dirichlet integral of $f$ at $\xi$ by

$$
D_{\xi}(f)=\int_{\mathbb{T}}\left|\frac{f(\eta)-f(\xi)}{\eta-\xi}\right|^{2} d m(\eta),
$$

and set $D_{\xi}(f)=\infty$ if $f(\xi)$ does not exist. See [9] for more information about the local Dirichlet integral.

A formula of J. Douglas [3] for the Dirichlet integral shows that

$$
D(f)=\int_{\mathbb{T}} D_{\xi}(f) d m(\xi),
$$

which implies that $D_{\xi}(f) \in L^{1}(\mathbb{T})$ whenever $f \in \mathcal{D}$.

For $f, g \in \mathcal{D}$ and $\xi \in \mathbb{T}$ such that both $D_{\xi}(f)$ and $D_{\xi}(g)$ are finite, define

$$
D_{\xi}(f, g)=\int_{\mathbb{T}} \frac{(f(\eta)-f(\xi)) \overline{(g(\eta)-g(\xi))}}{|\eta-\xi|^{2}} d m(\eta),
$$

which is called the mixed local Dirichlet integral of $f$ and $g$ at $\xi$ [11]. Then $D_{\xi}(f, g) \in L^{1}(\mathbb{T})$ and $D_{\xi}(f)=D_{\xi}(f, f)$.

The following lemma comes from [11, Lemma 2.1]. 
Lemma 2.4. Let $f, g \in \mathcal{D}, \lambda \in \mathbb{D}$, and $\alpha, \beta \in \mathbb{C}$. Then

$$
\begin{aligned}
\left\langle\frac{1}{1-\bar{\lambda} z} f, g\right\rangle= & \int_{\mathbb{T}} \frac{1}{1-\bar{\lambda} \xi}\left(f(\xi) \overline{g(\xi)}+D_{\xi}(f, g)\right) d m(\xi) \\
& +\int_{\mathbb{T}} \frac{\bar{\lambda} \xi}{(1-\bar{\lambda} \xi)^{2}} f(\xi) \overline{g(\xi)} d m(\xi)
\end{aligned}
$$

and

$$
\begin{aligned}
& \int_{\mathbb{T}} \frac{\bar{\lambda} \xi}{(1-\bar{\lambda} \xi)^{2}} f(\xi) \overline{g(\xi)} d m(\xi) \\
& \quad=\int_{\mathbb{T}} \frac{\bar{\lambda} \xi}{(1-\bar{\lambda} \xi)^{2}}(f(\xi)-\alpha) \overline{(g(\xi)-\beta)} d m(\xi)+\alpha \overline{\lambda g^{\prime}(\lambda)} .
\end{aligned}
$$

Proof of Theorem 1.1. The proof will make use of some ideas of the proof of [11, Lemma 3.1].

Let $U: \mathcal{M}_{1} \rightarrow \mathcal{M}_{2}$ be a unitary operator such that $\left.U M_{z}\right|_{\mathcal{M}_{1}}=\left.M_{z}\right|_{\mathcal{M}_{2}} U$. Let $\varphi \in \mathcal{M}_{1} \ominus z \mathcal{M}_{1}$ and $\psi=U \varphi$. Then it is easy to verify that $\psi \in$ $\mathcal{M}_{2} \ominus z \mathcal{M}_{2}$. By Lemma 2.3,

$$
|\varphi(\xi)|^{2}=|\psi(\xi)|^{2}, \quad \text { a.e. } \xi \in \mathbb{T} .
$$

Since $\left.U M_{z}\right|_{\mathcal{M}_{1}}=\left.M_{z}\right|_{\mathcal{M}_{2}} U$, for $\lambda \in \mathbb{D}$ we have

$$
\left\langle\frac{1}{1-\bar{\lambda} z} \varphi, \varphi\right\rangle=\left\langle\frac{1}{1-\bar{\lambda} z} \psi, \psi\right\rangle \text {. }
$$

By (2.1),

$$
\begin{aligned}
& \int_{\mathbb{T}} \frac{1}{1-\bar{\lambda} \xi}\left(|\varphi(\xi)|^{2}+D_{\xi}(\varphi)\right) d m(\xi)+\int_{\mathbb{T}} \frac{\bar{\lambda} \xi}{(1-\bar{\lambda} \xi)^{2}}|\varphi(\xi)|^{2} d m(\xi) \\
& =\int_{\mathbb{T}} \frac{1}{1-\bar{\lambda} \xi}\left(|\psi(\xi)|^{2}+D_{\xi}(\psi)\right) d m(\xi)+\int_{\mathbb{T}} \frac{\bar{\lambda} \xi}{(1-\bar{\lambda} \xi)^{2}}|\psi(\xi)|^{2} d m(\xi) .
\end{aligned}
$$

By (2.3) and (2.4), we obtain

$$
\int_{\mathbb{T}} \frac{1}{1-\bar{\lambda} \xi} D_{\xi}(\varphi) d m(\xi)=\int_{\mathbb{T}} \frac{1}{1-\bar{\lambda} \xi} D_{\xi}(\psi) d m(\xi) .
$$

Taking $\lambda=0$ in (2.5), we have

$$
\int_{\mathbb{T}} D_{\xi}(\varphi) d m(\xi)=\int_{\mathbb{T}} D_{\xi}(\psi) d m(\xi) .
$$

Since the Poisson kernel is

$$
P_{\lambda}(\xi)=\frac{1-|\lambda|^{2}}{|1-\bar{\lambda} \xi|}=2 \operatorname{Re}\left(\frac{1}{1-\bar{\lambda} \xi}\right)-1,
$$


by (2.5) and (2.6) we get

$$
\int_{\mathbb{T}} P_{\lambda}(\xi) D_{\xi}(\varphi) d m(\xi)=\int_{\mathbb{T}} P_{\lambda}(\xi) D_{\xi}(\psi) d m(\xi) .
$$

Since $D_{\xi}(\varphi), D_{\xi}(\psi) \in L^{1}(\mathbb{T})$, it follows that

$$
D_{\xi}(\varphi)=D_{\xi}(\psi), \quad \text { a.e. } \xi \in \mathbb{T} .
$$

On the other hand, by (2.3), we have

$$
\int_{\mathbb{T}} \frac{\bar{\lambda} \xi}{(1-\bar{\lambda} \xi)^{2}}|\varphi(\xi)|^{2} d m(\xi)=\int_{\mathbb{T}} \frac{\bar{\lambda} \xi}{(1-\bar{\lambda} \xi)^{2}}|\psi(\xi)|^{2} d m(\xi) .
$$

Then by (2.2), for $\alpha, \beta \in \mathbb{C}$,

$$
\begin{aligned}
& \int_{\mathbb{T}} \frac{\bar{\lambda} \xi}{(1-\bar{\lambda} \xi)^{2}}|\varphi(\xi)-\alpha|^{2} d m(\xi)+\alpha \overline{\lambda \varphi^{\prime}(\lambda)} \\
&=\int_{\mathbb{T}} \frac{\bar{\lambda} \xi}{(1-\bar{\lambda} \xi)^{2}}|\psi(\xi)-\beta|^{2} d m(\xi)+\beta \overline{\lambda \psi^{\prime}(\lambda)} .
\end{aligned}
$$

In particular, for $\eta \in \mathbb{T}$ such that $\varphi(\eta), \psi(\eta), \varphi^{\prime}(\eta), \psi^{\prime}(\eta), D_{\eta}(\varphi)$ and $D_{\eta}(\psi)$ are finite, taking $\alpha=\varphi(\eta)$ and $\beta=\psi(\eta)$, we have

$$
\begin{aligned}
\int_{\mathbb{T}} \frac{\bar{\lambda} \xi|\xi-\eta|^{2}}{(1-\bar{\lambda} \xi)^{2}} & \frac{|\varphi(\xi)-\varphi(\eta)|^{2}}{|\xi-\eta|^{2}} d m(\xi)+\varphi(\eta) \overline{\lambda \varphi^{\prime}(\lambda)} \\
& =\int_{\mathbb{T}} \frac{\bar{\lambda} \xi|\xi-\eta|^{2}}{(1-\bar{\lambda} \xi)^{2}} \frac{|\psi(\xi)-\psi(\eta)|^{2}}{|\xi-\eta|^{2}} d m(\xi)+\psi(\eta) \overline{\lambda \psi^{\prime}(\lambda)} .
\end{aligned}
$$

Since

$$
\frac{\bar{\lambda} \xi|\xi-\eta|^{2}}{(1-\bar{\lambda} \xi)^{2}} \rightarrow-1 \quad \text { as } \lambda \rightarrow \eta,
$$

letting $\lambda \rightarrow \eta$ nontangentially, it follows from (2.8) and the dominated convergence theorem that

$$
-D_{\eta}(\varphi)+\varphi(\eta) \overline{\eta \varphi^{\prime}(\eta)}=-D_{\eta}(\psi)+\psi(\eta) \overline{\eta \psi^{\prime}(\eta)} .
$$

Combining this with (2.7), we have

$$
\varphi(\eta) \overline{\eta \varphi^{\prime}(\eta)}=\psi(\eta) \overline{\eta \psi^{\prime}(\eta)}, \quad \text { a.e. } \eta \in \mathbb{T} \text {. }
$$

From (2.3) and (2.9), we get

$$
\frac{\varphi^{\prime}(\xi)}{\varphi(\xi)}=\frac{\psi^{\prime}(\xi)}{\psi(\xi)}, \quad \text { a.e. } \xi \in \mathbb{T} .
$$

By Theorem 2.2(ii), both $\varphi^{\prime}$ and $\psi^{\prime}$ are in $N(\mathbb{D})$, and hence combining (2.10) and a simple reasoning shows that

$$
\frac{\varphi^{\prime}(z)}{\varphi(z)}=\frac{\psi^{\prime}(z)}{\psi(z)}, \quad z \in \mathbb{D}
$$


This implies that $\varphi=c \psi$ for some constant $c$. By Theorem 2.1, $\mathcal{M}_{1}=\mathcal{M}_{2}$ as desired.

3. Unitary equivalence of invariant subspaces in the norm $\|\cdot\|_{0}$. In this section, we consider the Dirichlet space $\mathcal{D}$ in the norm $\|\cdot\|_{0}$,

$$
\|f\|_{0}^{2}=|f(0)|^{2}+D(f), \quad f \in \mathcal{D} .
$$

We use $\langle,\rangle_{0}$ to denote the corresponding inner product.

We begin with a discussion of Carleson's formula. Let $f=B S F$ be the canonical factorization of $f$, where $B=\prod_{j=1}^{\infty} \frac{\bar{a}_{j}}{\left|a_{j}\right|} \frac{a_{j}-z}{1-\bar{a}_{j} z}$ is a Blaschke product, $S$ is the singular part of $f$, and $\mu$ the corresponding singular measure, and $F$ is the outer part of $f$. Then Carleson's formula [2] (see also [9, Corollary 3.6]) is

$$
\begin{aligned}
D(f)= & \frac{1}{2 \pi} \int_{0}^{2 \pi} \sum_{n=1}^{\infty} P_{\alpha_{n}}\left(e^{i s}\right)\left|f\left(e^{i s}\right)\right|^{2} d s \\
& +\frac{1}{2 \pi} \int_{0}^{2 \pi} \int_{0}^{2 \pi} \frac{2}{\left|e^{i t}-e^{i s}\right|^{2}} d \mu(t)\left|f\left(e^{i s}\right)\right|^{2} d s \\
& +\frac{1}{2 \pi} \int_{0}^{2 \pi} \frac{1}{2 \pi} \int_{0}^{2 \pi} \frac{\left(e^{2 u\left(e^{i t}\right)}-e^{2 u\left(e^{i s}\right)}\right)\left(u\left(e^{i t}\right)-u\left(e^{i s}\right)\right)}{\left|e^{i t}-e^{i s}\right|^{2}} d t d s
\end{aligned}
$$

for $f \in \mathcal{D}$, where $u\left(e^{i t}\right)=\log \left|f\left(e^{i t}\right)\right|$, and $P_{\alpha}\left(e^{i t}\right)$ is the Poisson kernel.

By Carleson's formula, it is easy to verify that

$$
\|z f\|_{0}^{2}=D(z f)=\|f\|_{2}^{2}+D(f)=\|f\|^{2}, \quad f \in \mathcal{D} .
$$

Proof of Theorem 1.2. By Theorem 1.1, it suffices to prove that if $\mathcal{M}_{1}$ and $\mathcal{M}_{2}$ are unitarily equivalent in the norm $\|\cdot\|_{0}$, then they are unitarily equivalent in the norm $\|\cdot\|$.

Let $V: \mathcal{M}_{1} \rightarrow \mathcal{M}_{2}$ be a unitary operator such that $\left.V M_{z}\right|_{\mathcal{M}_{1}}=\left.M_{z}\right|_{\mathcal{M}_{2}} V$. For $f \in \mathcal{M}_{1}$, let $g=V f$. It is enough to prove that

$$
\|f\|=\|g\| .
$$

Since $\left.V M_{z}\right|_{\mathcal{M}_{1}}=\left.M_{z}\right|_{\mathcal{M}_{2}} V$ and $V$ is unitary, we have $V(z f)=z g$ and

$$
\|z f\|_{0}=\|z g\|_{0} .
$$

By (3.1), the proof is complete.

4. Unitary equivalence of invariant subspaces in the norm $\|\cdot\|_{1}$. In this section, we consider the Dirichlet space $\mathcal{D}$ with the norm $\|\cdot\|_{1}$,

$$
\|f\|_{1}^{2}=\int_{\mathbb{D}}|f|^{2} d A+D(f), \quad f \in \mathcal{D} .
$$


Let $\langle\cdot, \cdot\rangle_{1}$ be the corresponding inner product. A direct computation shows that for nonnegative integers $n, m$ with $n \leq m$, and $f(z)=\sum_{l=0}^{\infty} a_{l} z^{l} \in \mathcal{D}$,

$$
\left\langle z^{n} f, z^{m} f\right\rangle_{1}=\sum_{k=m}^{\infty} a_{k-n} \bar{a}_{k-m}\left\langle z^{k}, z^{k}\right\rangle_{1}=\sum_{k=m}^{\infty} a_{k-n} \bar{a}_{k-m}\left(\frac{1}{k+1}+k\right) .
$$

Proof of Theorem 1.3. Let $f(z)=\sum_{l=0}^{\infty} a_{l} z^{l}$ be in $\mathcal{D}$ and $n, m$ be nonnegative integers with $n \leq m$. Then by (4.1),

$$
\begin{aligned}
\left\langle z^{n} f, z^{m} f\right\rangle_{1} & =\sum_{k=m}^{\infty} a_{k-n} \bar{a}_{k-m}\left(\frac{1}{k+1}+k\right), \\
\left\langle z^{n+1} f, z^{m+1} f\right\rangle_{1} & =\sum_{k=m+1}^{\infty} a_{k-(n+1)} \bar{a}_{k-(m+1)}\left(\frac{1}{k+1}+k\right) \\
& =\sum_{k=m}^{\infty} a_{k-n} \bar{a}_{k-m}\left(\frac{1}{k+2}+k+1\right),
\end{aligned}
$$

and similarly,

$$
\left\langle z^{n+2} f, z^{m+2} f\right\rangle_{1}=\sum_{k=m}^{\infty} a_{k-n} \bar{a}_{k-m}\left(\frac{1}{k+3}+k+2\right) .
$$

Taking $(4.2)-2 \times(4.3)+(4.4)$ yields

$$
\begin{aligned}
\left\langle z^{n} f, z^{m} f\right\rangle_{1}-2\left\langle z^{n+1} f, z^{m+1} f\right\rangle_{1}+ & \left\langle z^{n+2} f, z^{m+2} f\right\rangle_{1} \\
& =\sum_{k=m}^{\infty} \frac{2 a_{k-n} \bar{a}_{k-m}}{(k+1)(k+2)(k+3)} .
\end{aligned}
$$

Let $U: \mathcal{M}_{1} \rightarrow \mathcal{M}_{2}$ be a unitary operator such that $\left.U M_{z}\right|_{\mathcal{M}_{1}}=\left.M_{z}\right|_{\mathcal{M}_{2}} U$. Suppose $f(z)=\sum_{l=0}^{\infty} a_{l} z^{l}$ in $\mathcal{M}_{1}$ and $g(z)=\sum_{l=0}^{\infty} b_{l} z^{l}$ in $\mathcal{M}_{2}$ are such that $U f=g$. Then by (4.5), we have

$$
\sum_{k=m}^{\infty} \frac{a_{k-n} \bar{a}_{k-m}}{(k+1)(k+2)(k+3)}=\sum_{k=m}^{\infty} \frac{b_{k-n} \bar{b}_{k-m}}{(k+1)(k+2)(k+3)} .
$$

It is obvious that $\mathcal{D} \subset L_{a}^{2}\left(\left(1-|z|^{2}\right)^{2} d A(z)\right)$, the weighted Bergman space with the orthonormal basis $\left\{((k+1)(k+2)(k+3) / 2)^{1 / 2} z^{k}\right\}_{k=0}^{\infty}$, and a routine calculation shows that for $h(z)=\sum_{l=0}^{\infty} c_{l} z^{l}$ in $L_{a}^{2}\left(\left(1-|z|^{2}\right)^{2} d A(z)\right)$,

$$
\left\langle z^{n} h, z^{m} h\right\rangle_{L_{a}^{2}\left(\left(1-|z|^{2}\right)^{2} d A(z)\right)}=\sum_{k=m}^{\infty} \frac{2 c_{k-n} \bar{c}_{k-m}}{(k+1)(k+2)(k+3)} .
$$

By (4.6) and (4.7), for $f \in \mathcal{M}_{1}$ and $g \in \mathcal{M}_{2}$ with $U f=g$,

$$
\left\langle z^{n} f, z^{m} f\right\rangle_{L_{a}^{2}\left(\left(1-|z|^{2}\right)^{2} d A(z)\right)}=\left\langle z^{n} g, z^{m} g\right\rangle_{L_{a}^{2}\left(\left(1-|z|^{2}\right)^{2} d A(z)\right)} .
$$


This means that $[f]$ and $[g]$, the invariant subspaces of $L_{a}^{2}\left(\left(1-|z|^{2}\right)^{2} d A(z)\right)$ generated by $f$ and $g$, respectively, are unitarily equivalent. Therefore by Theorem 1 in [7], $g=c f$ for some constant $c$. This implies that $\mathcal{M}_{1}=\mathcal{M}_{2}$, completing the proof.

Acknowledgements. The author thanks the referee for pointing out equation (3.1), which shortened the proof of Theorem 1.2 significantly.

This work was partially supported by NSFC (10525106) and NKBRPC (2006CB805905). The research of L. K. Zhao was also partially supported by NSFC (10971195).

\section{References}

[1] R. Adams and J. Fournier, Sobolev Spaces, 2nd ed., Academic Press, Amsterdam, 2003 .

[2] L. Carleson, A representation formula for the Dirichlet integral, Math. Z. 73 (1960), 190-196.

[3] J. Douglas, Solution of the problem of Plateau, Trans. Amer. Math. Soc. 33 (1931), 263-321.

[4] J. Garnett, Bounded Analytic Functions, Academic Press, New York, 1981.

[5] S. Richter, Invariant subspaces of the Dirichlet shift, J. Reine Angew. Math. 386 (1988), 205-220.

[6] -, A representation theorem for cyclic analytic two-isometries, Trans. Amer. Math. Soc. 328 (1991), 325-349.

[7] - Unitary equivalence of invariant subspaces of Bergman and Dirichlet space, Pacific J. Math. 133 (1988), 151-155.

[8] S. Richter and A. Shields, Bounded analytic functions in the Dirichlet space, Math. Z. 198 (1988), 151-159.

[9] S. Richter and C. Sundberg, A formula for the local Dirichlet integral, Michigan Math. J. 38 (1991), 355-379.

[10] —, -, Multipliers and invariant subspaces in the Dirichlet space, J. Operator Theory 28 (1992), 167-186.

[11] - - - Invariant subspaces of the Dirichlet shift and pseudocontinuations, Trans. Amer. Math. Soc. 341 (1994), 863-879.

[12] W. Ross, The classical Dirichlet space, in: Recent Advances in Operator-Related Function Theory, Contemp. Math. 393, Amer. Math. Soc., Providence, RI, 2006, 171-197.

Kunyu Guo

School of Mathematics Sciences

Fudan University

Shanghai, 200433, P.R. China

E-mail: kyguo@fudan.edu.cn
Liankuo Zhao (corresponding author) School of Mathematics and Computer Sciences Shanxi Normal University Linfen, 041004, P.R. China E-mail: lkzhao@sxnu.edu.cn 